\title{
SINTOM AS DEPRESSIVOS E ANSIEDADE EM PACIENTES COM CEFALÉIA DO TIPO TENSIONAL CRÔNICA E EPISÓDICA
}

\author{
André Palma da Cunha Mattaㄹ ${ }^{1}$ Pedro F. Moreira Filho²
}

\begin{abstract}
RESUMO - Introdução: A cefaléia do tipo tensional (CTT), dor cefálica mais comum, tem mecanismos fisiopatológicos, epidemiologia e apresentação clínica pouco estudados. Depressão e ansiedade são comorbidades especialmente enfocadas entre seus portadores. Objetivo: Estudar a ocorrência de sintomas depressivos e ansiedade entre portadores de CTT episódica (CTTE) e crônica (CTTC). Metodologia: Cinqüenta pacientes com CTTE e cinqüenta com CTTC preencheram os inventários de depressão e de ansiedade de Beck. Somente foram considerados aqueles com escores moderado ou grave. Resultados: Entre portadores de CTTE, ansiedade e sintomas depressivos foram encontrados em 60 e $32 \%$ dos pacientes, respectivamente. Em pacientes com CTTC, ansiedade foi detectada em $44 \%$ e sintomas depressivos foram observados em $40 \%$ da amostra. Conclusão: ambas as comorbidades são importantes em pacientes com CTT. Negligenciá-las significa risco de falência no tratamento, contribuindo para queda na qualidade de vida .
\end{abstract}

PALAVRAS-CHAVE: cefaléia do tipo tensional episódica, cefaléia do tipo tensional crônica, depressão, ansiedade.

\section{Depressive symptoms and anxiety in patients with chronic and episodic tension-type headache}

\begin{abstract}
Background: Tension-type headache $(T T H)$ is recognized as the most prevalent type of headache. Despite this, there is a limited understanding of the entity's physiology, epidemiology, and clinical presentation. Anxiety and depression are recognized comorbidities present among patients with $\mathrm{TTH}$. Objective: To quantify the prevalence of anxiety and depression among patients with episodic and chronic $\mathrm{TTH}$. Method: fifty patients with episodic TTH and fifty patients with chronic TTH completed Beck's anxiety and depression surveys. Only patients presenting with moderate to severe scores were considered. Results: among the patients with episodic $\mathrm{TTH}$, anxiety and depression were observed in $30(60 \%)$ and $16(32 \%)$ patients respectively. Among the patients with chronic TTH, anxiety was observed in 22 (44\%) patients, and depression was observed in 20 (40\%). Conclusion: both comorbidities are important among patients with episodic and chronic $\mathrm{TTH}$. Neglecting this association may result in failure of symptomatic and prophylactic treatment ultimately leading to lost quality of life.
\end{abstract}

KEY WORDS: episodic tension-type headache, chronic tension-type headache, depression, anxiety.

A cefaléia do tipo tensional (CTT) caracteriza-se, classicamente, por dor de caráter constritivo (não pulsátil), geralmente bilateral, de intensidade leve a moderada, não agravada por atividades físicas de rotina e com duração variável entre trinta minutos e sete dias ${ }^{1,2}$. Seus critérios diagnósticos atuais foram estabelecidos em 1988 pelo Headache Classification Committe of The International Headache Society². Embora seja reconhecida como a forma mais comum de dor cefálica, éainda muito pouco estudada. Escassos são os conhecimentos acerca de seus mecanismos fisiopatológicos ${ }^{3}$, seus dados epidemiológicos, suas formas de apresentação e seu tratamento farmacológico ${ }^{4}$. Em diversos estudos clínicos e epidemiológicos, duas comorbidades são especialmente enfocadas entre pacientes com CTT: depressão eansiedade. Estas condições são mais freqüentes e graves entre os portadores de cefaléia do que na população geral, principalmente se considerarmos enfermos com CTT crônica (CTTC), cefaléia mista (CTT e migrânea) e cefaléia por abuso de analgésicos ${ }^{5,6}$.

A ocorrência de sintomas depressivos e ansiedade em pacientes com CTT é analisada neste estudo.

Universidade Federal Fluminense ( UFF ) Niterói RJ, Brasil: Serviço de Neurologia Hospital Universitário Antônio Pedro ( HUAP): ${ }^{1}$ Mestre em Neurologia pela UFF; ²Doutor em Neurologia, Professor Adjunto 4 do Serviço de Neurologia da UFF, Chefe do Serviço de Investigação e Tratamento das Cefaléias do HUAP.

Recebido 12 Março 2003, recebido na forma final 23 Junho 2003. Aceito 11 Julho 2003.

Dr. André Palma da Cunha Matta - Rua Rabino Henrique Lemle 389/202 - 22790-040 Rio de Janeiro RJ - Brasil.

E-mail: andrepcmatta@ hotmail.com; pmoreira@ ism.com.br 


\section{METODO}

No período de março de 1999 a março de 2000, foram avaliados no Ambulatório de Cefaléias do Serviço de Neurologia do Hospital Universitário Antônio Pedro (HUAP), 50 pacientes com CTIE e 50 com CTTC. A maioria, $80 \%$ dos portadores de CTTC e $60 \%$ dos portadores deCTTE, teve demanda espontânea, sendo os demais referidos ao ambulatório de cefaléias provenientes das redes pública e privada ou de outros setores do HUAP.

Foram adotados como critérios de inclusão: idade acima de 18 anos; exame neurológico normal; exames complementares(quando necessários) normais, incluindo tomografia computadorizada ou ressonância magnética do encéfalo; ausência de passado migranoso ou critérios diagnósticos atuais para migrânea ou outras cefaléias primárias ou secundárias; preenchimento dos critérios diagnósticos para CTTC ou CTTE conforme o Headache Classification Committe of The International Headache Society, $1988^{2}$.

Todos os pacientes foram entrevistados e examinados por um dos autores. Para avaliar a presença de sintomas depressivos e de ansiedade, os enfermos preencheram os inventários de depressão e de ansiedade de Beck ${ }^{7}$. Foram considerados portadores destas condições aqueles com escores de moderado a grave.

O estudo foi previamente aprovado pela Comissão de Ética que regulamenta a produção científica no Serviço de Neurologia do HUAP e, para serem incluídos no estudo, os pacientes forneceram, por ocasião da primeira entrevista, o consentimento informado.

Os dados obtidos foram analisados através do teste $t$ e do teste do qui-quadrado para se verificar seu valor estatístico, adotando-se $p<0,05$ como parâmetro de significância.

\section{RESULTADOS}

Dos 50 pacientes com CTTE estudados, 40 (80\%) eram do gênero feminino e 10 (20\%) do masculino, obtendo-se uma relação 4:1 entre mulheres e homens. Entre os portadores de CTTC, 39 eram do gênero feminino (78\%) e 11 do masculino ( $22 \%$ ), obtendo-se uma razão mulheres / homens de 3,5: 1. Entre pacientes com CTTE, a idade por ocasião da pesquisa variou entre 18 e 60 anos, com média de 30 anos. Nos pacientes com CTTC, a idade variou entre 30 e 60 anos, com média de 42,8 anos .

Nos pacientes com CTTE, a ansiedade e os sintomas depressivos, conforme critérios já mencionados, foram observados em 30 (60 \%) e em 16 pacientes (32\%), respectivamente (Tabela 1 ). Neste grupo, não houve diferença estatística significativa quanto ao gênero ou faixa etária para ambas comorbidades ( $p=0,110$ e $p=0,210$, respectivamente).

A ansiedade foi detectada em 22 pacientes (44 \%) com CTTC; já os sintomas depressivos foram constatados em 20 pacientes (40\%)(Tabela 2). Mais uma vez, não houve diferença significativa entre os sexos na prevalência de ansiedade e sintomas depressivos $(p=0,250)$. Houve tendência, embora não significativa do ponto de vista estatístico, de prevalecer a ansiedade numa faixa etária menor do que os sintomas depressivos $(p=0,070)$.

Através do testet, não foi possível identificar diferença estatística significativa para a ocorrência de depressão e ansiedade nos dois grupos $(p=0,410 \mathrm{e}$ $p=0,112$, respectivamente).

\section{DISCUSSÃO}

A CTT foi descrita anteriormente como cefaléia de origem psicogênica. Há muito se reconhece a associação entre quadros de ansiedade e depressão e esta modalidade de dor. O estado álgico muscular e o aumento do tônus da musculatura pericraniana, ambos implicados na patogênese da CTT, foram classicamente atribuídos à "reação individual ao estres$\mathrm{se}^{\prime 8}$. A depressão é, uma síndrome clínica e não uma entidade única. Pode ser dividida, segundo sua etiologia, em reacional ou endógena; de acordo com sintomas associados, em neurótica ou psicótica e, por seu curso evolutivo, em uni ou bipolar, senil e involutiva ${ }^{8}$. Independente da forma como é classificada, a depressão é freqüentemente abordada em trabalhos que estudam a dor crônica. Em nosso meio, podemos citar o estudo realizado por Martinez e colaboradores, que encontraram percentual significativo de depressão $(80,0 \%)$ em 47 mulheres com fibromialgia9. Em nosso estudo, restringimo - nos a detectar a presença de sinais ou sintomas depressivos, evitando, portanto, firmar diagnóstico de depressão entre os pacientes entrevistados.

Tabela 1. Ansiedade e sintomas depressivos entre 50 portadores de CTTE.

\begin{tabular}{llcc}
\hline Comorbidade & presente & ausente & total \\
\hline Ansiedade & $30(60 \%)$ & $20(40 \%)$ & $50(100 \%)$ \\
Sintomas & $16(32 \%)$ & $34(68 \%)$ & $50(100 \%)$ \\
depressivos & & & \\
\hline
\end{tabular}

Tabela 2. Ansiedade e sintomas depressivos entre 50 portadores de CTTC.

\begin{tabular}{llcc}
\hline Comorbidade & presente & ausente & total \\
\hline Ansiedade & $22(44 \%)$ & $28(56 \%)$ & $50(100 \%)$ \\
Sintomas & $20(40 \%)$ & $30(60 \%)$ & $50(100 \%)$ \\
Depressivos & & & \\
\hline
\end{tabular}


A prevalência de sintomas depressivos na população em geral está em torno de $6 \%^{10}$. Do ponto de vista fisiopatológico, propõe-se que a proximidade existente entre a CTT e a depressão tenha origem na disfunção de vias serotoninérgicas, presente nestas duas condições ${ }^{11}$. Doentes com CTT apresentam níveis plasmáticos e concentração plaquetária de serotonina reduzidos ${ }^{12}$. Existe captação maior de serotonina nas plaquetas de portadores de CTTC quando comparados a migranosos e à população em geral, o que pode refletir, no primeiro grupo, vigência de estado carencial ${ }^{13}$. No entanto, não está claro se estas alterações refletem distúrbios periféricos ou centrais do metabolismo da serotonina ${ }^{6}$. O que se sabe, até o momento, é que medicamentos que atuam nas vias serotoninérgicas centrais (sertralina, por exemplo) podem causar alívio da dor nos doentes com CTT ${ }^{14}$.

Estudo recente, realizado no Equador, país com contexto sócio - econômico semelhante ao nosso, revelou a presença de sintomas depressivos em $33,7 \%$ de 89 portadores de CTTC ${ }^{8}$. Em nossa amostra encontramos sintomas depressivos em $40 \%$ dos pacientes com CTTC, o que nos aproxima dos resultados do estudo equatoriano. Entretanto, entre portadores de CTTE, a presença destes sintomas foi relativamente alta, com $32 \%$ da nossa amostra. Como pouco se tem publicado acerca desta associação (depressão e CTTE), não dispomos de dados significativos na literatura para comparação. Acreditamos que fatores ambientais bastante significativos em nossa casuística, como desemprego, alcoolismo, baixos salários, descontentamento com a situação profissional e elevada prevalência de ansiedade possam ter contribuído para este achado.

A ansiedade também pode ser considerada uma síndrome, com sinais e sintomas bem definidos. Diversas desordens psiquiátricas têm como pano de fundo a ansiedade; destas as mais frequentes no contexto de cefaléia são: distúrbio somatoforme (histeria), transtorno obsessivo - compulsivo e crise de ansiedade generalizada ${ }^{8}$. Em nosso estudo, encontramos alta prevalência de ansiedade (60\%) entre doentes com CTTE e uma moderada prevalência (44\%) entre aqueles com CTTC, não havendo diferença estatística significativa entre os dois grupos $(p=0,112)$. Os valores, entretanto, encontram-se mais elevados dos que os referidos na literatura em geral. No mesmo estudo equatoriano mencionado anteriormente, observou-se que $64 \%$ dos casos de CTT não se relacionavam com o estresse ${ }^{8}$. Talvez esta discrepância se deva a diferenças metodológicas, uma vez que o inventário de ansiedade de Beck, adotado em nosso estudo, tem elevada sensibilidade para detectar a presença desta condição. Mais uma vez, características sócio - econômicas da amostra podem ter colaborado para esses achados conflitantes. Deve ser valorizado como possível fator ansiogênico o receio freqüentemente relatado pelos pacientes de serem, portadores de patologias graves, como tumores cerebrais ou doenças cerebrovasculares. Este dado condiz com achados da literatura, que mostram que $77 \%$ dos pacientes esperam de quem os atende, em primeiro lugar, saber sobre a etiologia da sua dor, excluindo-se assim causas potencialmente graves ${ }^{15}$.

Admite-se que doentes com CTTE apresentem sintomas de ansiedade mais intensos, enquanto aqueles com CTTC têm sintomas depressivos mais exuberantes ${ }^{6,16,17}$. Tanto a ansiedade quanto a depressão podem atuar em nível central como facilitadores das aferências dolorosas, participando desta forma da patogênese da dor (especialmente na CTTC) ${ }^{16}$.

Estudo semelhante ao nosso, realizado na Coréia, em que 49 pacientes com CTTC (entre outros) foram avaliados através do inventário de depressão de Beck, também não encontrou correlação precisa entre a gravidade dos sintomas depressivos e a idade, a duração, o caráter, a intensidade e a freqüência da dor ${ }^{17}$.

Se a depressão predomina em faixa etária média de 40 anos e no gênero feminino ${ }^{18}$ e se a ansiedade acontece com maior freqüência no final da adolescência e no início da idade adulta predominando também no gênero feminino ${ }^{19}$, como explicar então que entre pacientes com CTT não se repita este padrão? Não é de se surpreender que encontremos achados diversos daqueles observados na população em geral para prevalência de depressão porque lidamos com um grupo selecionado de indivíduos, com características peculiares de personalidade, humor, reação ao estresse e, sobretudo, de funcionamento bioquímico cerebral. Acreditamos que o mesmo argumento seja válido para a ansiedade.

\section{CONCLUSÃO}

Depressão e ansiedade são comorbidades importantes em pacientes com CTT. Negligenciá-las pode significar falência no tratamento sintomático ou profilático e contribuir para uma qualidade de vida ruim entre seus portadores ${ }^{16}$. 


\section{REFERÊNCIAS}

1. Rasmunssen $B K$, Jensen $R$, Olesen J. A population-based analysis of the diagnostic criteria of the International Headache Society. Cephalalgia 1991;11:129-134.

2. Headache Classification Committe of the International Headache Society. Classification and diagnostic criteria for headache disorders, cranial neuralgias, and facial pain. Cephalalgia 1988;8:1-96.

3. Vandenheede M, Schoenen J. Central mechanisms in tension-type headaches. Curr Pain Headache Rep 2002;6:392-400.

4. Stillman MJ. Pharmacotherapy of tension: type headache . Curr Pain Headache Rep 2002;6:408-413.

5. Mitsikostas DD. Comorbity of headache and depressive disorders. Cephalalgia 1999;19:211-217.

6. Kunkel RS. Diagnóstico e tratamento das cefaléias por contração muscular (tipo tensão). Clin Med Am N orte 1991;3:613-621.

7. Matta APC. Cefaléia do tipo tensional: estudo clínico. Dissertação de Mestrado Universidade Federal Fluminense, Rio de Janeiro Niterói, 2001.

8. Serrano-Dueñas M. Cefalea tipo tensional crónica y depresión. Rev Neurol 2000;30:822-826.

9. Martinez JE, Ferraz MB, Fontana AM, A tra E. Psychological aspects of Brazilian women with fibromyal gia. J Psychosom Res 1995;39:167-174.
10. Breslin NA. Clinical syndromes. In Wiener JM, Breslin NA (eds). The behavioral sciences in psychiatry. 3.Ed. Philadelphia: Williams and Wilkins, 1995:259-277.

11. Silbertein SD. Headachein dinical practice. Oxford: Oxford Univ Press, 1998:91-100.

12. Antonhy M. Plasma serotonin in platelets with chronic tension headaches. J Neurol Neurosurg Psychiatry 1989;52:182-184.

13. Shukla R. Serotonin in tension headache.J Neurol Neurosurg Psychiatry 1987;50:1682-1684.

14. Singh NN, Misra S. Sertralinein chronic tension-typeheadache. J Assoc Physicians India 2002;50:873-878.

15. Pakard RC. What does the patient want? Headache 1979;19:370-375.

16. Yucel B, KoraK, Ozyalcin S, A lcalar N, Ozdemir O, Yucel A. Depression, automatic thoughts, alexithymia, and assertiveness in patients with tension-type headache. Headache 2002;42:194-199.

17. Choi YC, Kim WJ, Kim CH, Lee MS. A clinical study of chronic headaches: clinical characteristics and depressive trends in migraine and tension-type headache.Yonsei Med J 1995;36:508-514.

18. Kaplan HI, Sadock BJ. Manual de psiquiatria clínica 2.Ed. trad. Batista D. Porto Alegre: Artes Médicas,1998:109-124.

19. Kaplan HI, Sadock BJ. Manual de psiquiatria clínica. 2.Ed. trad. Batista D. Porto Alegre: Artes Médicas,1998:125-137. 